\title{
Woe of Pregnant Females: The Seductive Mona Lisa Syndrome
}

Virendra Atam, Amit Shankar Singh, Liza Das, Laxmi Shankar Singh

From the Department of Medicine, King George's Medical University,

Uttar Pradesh, Lucknow, India.

\section{Abstract:}

Mona Lisa syndrome is the most fascinating of all pregnancy related syndromes. It represents the characteristic idiopathic lower motor neuron facial nerve palsy mainly presenting in third trimester and associated with facial asymmetry caused by protracted and incomplete motor regeneration of the facial nerve. We encountered a pregnant female with idiopathic left facial nerve palsy of initial House-Brackmann Grade VI who on serial monitoring with facial electroneurography was identified as Mona Lisa syndrome.

Key words: Facial Nerve, Facial Paralysis, Bell Palsy, Motor Neurons, Pregnancy, Syndrome.

\section{Introduction}

Pregnancy induces several biochemical and physiological changes in the body which culminate into diseases or syndrome specific for pregnancy. One of them is the idiopathic facial nerve palsy. A distinguishing feature in few patients is facial muscle contracture leading to abnormal expressions that develops subsequent to Bell's palsy, when the facial nerve has undergone partial Wallerian degeneration and has regenerated. Though it is a plight for pregnant females, but its name is linked to a very fascinating Mona Lisa syndrome after the renounced French artist, mathematician, inventor, architect, and anatomist Leonardo da Vinci's marvelous piece of art "The Mona Lisa portrait," who was thought to be suffering from similar disease and her special facial expressions were contributed by this syndrome [1].

Here authors report a pregnant female of idiopathic left facial nerve palsy with sustained facial muscle contracture i.e. Mona Lisa syndrome presented during pregnancy.

\section{Case Report}

We here report a 27 year old rural pregnant female from India with 31 weeks pregnancy who presented with complains of sudden onset disfigurement of left side of face with disappearance of facial creases and nasolabial fold, unfurrowing of the forehead, and drooping of corner of the mouth. She had difficulty in closing left eyelids with sagging of lower lids, drooling of saliva and on attempted closure, upward rolling of eye on left side (Bell's phenomenon). Her symptoms progressed to maximum within two days of onset. She had no complains of any hearing loss, otorrhea, otalgia, vertigo, headaches, rashes, abnormal taste sensation or any

Corresponding Author: Dr. Amit Shankar Singh

Email: amitkgmumedicine@gmail.com

Received: November 30, 2013 | Accepted: December 24, 2013 | Published Online: December 30, 2013

This is an Open Access article distributed under the terms of the Creative Commons Attribution License (creativecommons.org/licenses/by/3.0)

Conflict of interest: None declared | Source of funding: Nil | DOl: http://dx.doi.org/10.17659/01.2013.0109 
other focal deficits. She had no associated medical illnesses (diabetes, autoimmune disorders, cancer), history of trauma (recent or remote), and previous surgery (otologic, rhytidectomy, parotidectomy). On examination patient had lower motor neuron type of House-Brackmann Grade VI facial palsy without affecting taste sensations and ability of hearing. A complete head and neck examination was performed, including otological examination of the ears, and a thorough neurological examination. No local pathology was detected in ears; parotid glands and rest of neurological examination was found to be normal.

Investigations revealed haemoglobin of $8.9 \mathrm{gm} \%$, with normal fasting glucose and $\mathrm{HbAlc}$ levels. There was no leucocytosis with total leukocyte counts of 5300 cells/cu.mm. Serum electrolytes, hepatic and renal functions were within normal limits. Enzyme-linked immunosorbent assay test to detect antibodies against Lyme disease bacteria and serum angiotensin-converting enzyme levels were negative. Magnetic Resonance Imaging of brain was without any apparent intracranial or extracranial abnormality. The audiometry tests were within the normal limits. On fourth day of illness electroneurography (ENoG) test was ordered and after supramaximal stimulation' baseline' response was obtained. The maximal stimulus (40 milliamperes) was required to obtain a 'plateau' response. The response obtained from the right side was 2140 microvolts $(\mu \mathrm{V})$. Using the same stimulation and recording parameters on the left side, the response obtained was $965 \mu \mathrm{V}$. The response was obtained and recorded from each side three times. Therefore, the response of the left side was determined to be $(965 / 2140) 45 \%$ of the normal side, also referred to as a ' $55 \%$ denervation' [Table 1].

The Guillain-Barré syndrome was excluded in this case as this characteristic disease presents with polyneuropathies with often bilateral involvement.
Similarly central nervous system lesions (e.g., multiple sclerosis, stroke, and tumor) present with supranuclear palsy (sparing of forehead) and additional neurologic symptoms which were not found in this case. Another important differential diagnosis Ramsay Hunt syndrome (an outbreak of herpes zoster in the facial nerve distribution) presents with prodrome of pain and often develops a vesicular eruption in the ear canal and pharynx. Infectious causes of facial nerve involvement like Lyme's disease ruled out as there was lack of history of tick exposure, rash, or arthralgias. Localized infections of ear canal like otitis media presents with gradual onset accompanied with ear pain, fever, and conductive hearing loss which were not present in this case. Diabetic microvascular neuropathy was ruled out as no past illness suggestive of diabetes mellitus was present and the patient had normoglycemic status. Sarcoidosis an important differential diagnosis, presents with polyneuropathies with bilateral involvement along with respiratory symptoms could be ruled out. Some localized salivary tumors compressing facial nerve was ruled by normal otologic and local area examination.

Artificial tears were used to lubricate the eyes to prevent corneal ulcerations. Oral corticosteroid therapy started to the patient at third day of illness. Oral prednisone was given in dose of $1 \mathrm{mg} / \mathrm{kg}$ or $60 \mathrm{mg} /$ day for 6 days, followed by a taper, for a total of 10 days. She did not respond well to the treatment and after 10 days she had HouseBrackmann facial nerve Grade 5. The second ENoG showed the right side amplitude at $2310 \mu \mathrm{V}$, and the left side was determined to be $1105 \mu \mathrm{V}$ 's. The response was determined to be (1105/2310) $48 \%$ of the normal side, or referred as $52 \%$ denervation [Table 1]. Patient was discharged subsequently and asked to come for follow-up.

Patient delivered a normal male baby by vaginal delivery in rural maternal child health care centre 
Table 1: Patient facial palsy grading by House-Brackmann Grade and facial electroneurography findings with duration.

\begin{tabular}{|c|c|c|c|}
\hline & \multicolumn{3}{|c|}{ Duration from onset of Left Facial Palsy } \\
\hline & Fourth Day & Tenth Day & Three Months \\
\hline $\begin{array}{l}\text { House-Brackmann } \\
\text { Grade of facial palsy }\end{array}$ & Grade 6 & Grade 5 & Grade 4 \\
\hline \multicolumn{4}{|l|}{ Facial Electroneurography } \\
\hline $\begin{array}{l}\text { Amplitude in microvolts } \\
\text { on normal (Right) side }\end{array}$ & 2140 & 2310 & 2275 \\
\hline $\begin{array}{l}\text { Amplitude in microvolts } \\
\text { on diseased (Left) side }\end{array}$ & 965 & 1105 & 1345 \\
\hline $\begin{array}{l}\text { Percent Response } \\
\text { (Amplitude on weak } \\
\text { side/Amplitude on normal side) }\end{array}$ & $45 \%$ & $48 \%$ & $59 \%$ \\
\hline $\begin{array}{l}\text { Percent Denervation } \\
\text { (100-Percent response) }\end{array}$ & $55 \%$ & $52 \%$ & $41 \%$ \\
\hline
\end{tabular}

of her village at 34 weeks gestation and finally visited in our department for follow up. She still had difficulty in closing left eye and drooling of saliva on left side of mouth along with abnormal facial expression when she shows some emotion due to asymmetry of her face. On examination she had House-Brackmann Grade 4 facial palsy on her left side [Fig.1]. The ENoG test revealed the right side amplitude of $2275 \mu \mathrm{V}$. The left side amplitude had improved to $1345 \mu \mathrm{V}$. The response was determined to be $(1345 / 2275) 59 \%$ of the normal side or referred as $41 \%$ denervation [Table 1]. Following these finding and her specific complains, Mona Lisa syndrome was suspected in this case. She was asked to continue artificial tears but facial nerve decompression was not tried.

\section{Discussion}

Idiopathic facial paralysis was first described by Sir Charles Bell (1774-1842) in the early 19th century and was named Bell's palsy after his name [2]. It was observed subsequently that women were affected two to four times more often than men of the same age, and pregnant women were affected three to four times more often than non-pregnant women [3]. Bell's palsy is believed to be caused by inflammation of the facial nerve at the geniculate

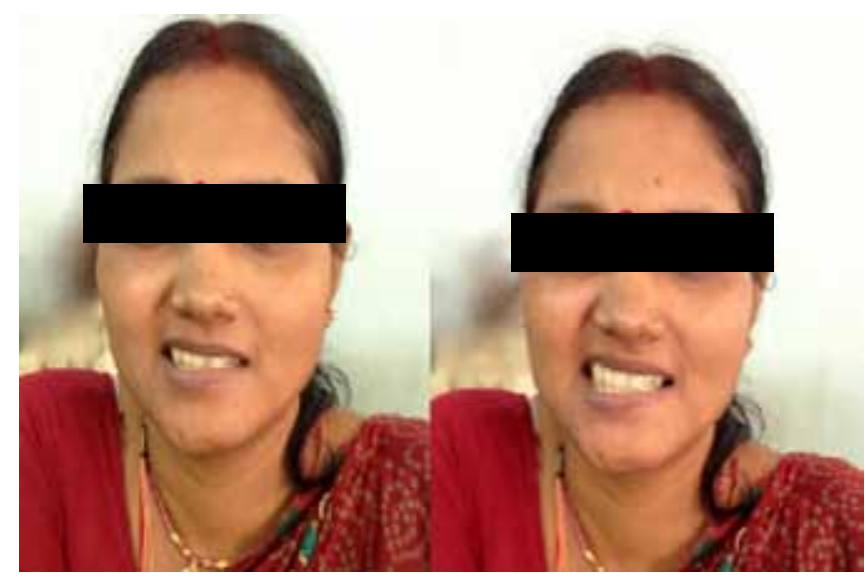

Fig.1: Postpartum female with facial asymmetry caused by protracted and incomplete motor regeneration of left facial nerve following idiopathic left facial nerve palsy (Mona Lisa syndrome). 
ganglion, which leads to compression and possible ischemia and demyelination. This ganglion lies in the facial canal at the junction of the labyrinthine and tympanic segments, where the nerve curves sharply toward the stylomastoid foramen. Classically, Bell's palsy has been defined as idiopathic, and the cause of the inflammatory process in the facial nerve remains uncertain. Increased extracellular fluid, viral inflammation, and the relative immune suppression of pregnancy are thought to be predisposing factors. A unique feature of reported cases of Bell's palsy in pregnancy is the presentation during the third trimester, or the immediate post-partum period $[4,5]$.

We also encountered a case of idiopathic facial nerve palsy but the sequence of events let us to a very fascinating Mona Lisa syndrome. Mona Lisa syndrome is idiopathic, acute peripheral facial nerve palsy in third trimester or postpartum period of pregnancy associated with facial muscle contracture developing when facial nerve has undergone partial Wallerian degeneration and has regenerated. The accompanying synkinesis would explain many of the known facts surrounding the riddle of smile in painting of Mona Lisa and is a classic example of Leonardo da Vinci as the compulsive anatomist who combined art and science. The enigmatic smile was merely the artist's rendering of the new mother's smile having been affected by Bell's palsy with partial recovery during her recent pregnancy. This concept for enigmatic smile of Mona Lisa's legendary portrait was first given by Adour who stressed more on facial asymmetry caused by protracted and incomplete motor regeneration of the nerve [1]. He also stated that some nerves grow along aberrant pathways resulting in synkinesis, leading to involuntary contraction of multiple facial muscles in the process of one or more voluntary motions. The initial management of this condition is not very different from idiopathic facial palsy but with late recovery and development of aberrant pathways may require other novel approaches like cosmetic surgical procedures or botulinum toxin (Botox) injections.

\section{Conclusion}

Pregnancy and postpartum periods are vulnerable to develop idiopathic facial nerve palsy. Partial recovery from weakness during pregnancy or postpartum period following facial nerve palsy gives clue for Mona Lisa syndrome. Though a fancy name, Mona Lisa syndrome is an agony for suffering female restraining her indigenous expressions along with physical turmoil

\section{References}

1. Adour KK. Mona Lisa syndrome: solving the enigma of the Gioconda smile. Ann Otol Rhinol Laryngol. 1989;98:196-199.

2. Bell C. The Nervous System of the Human Body. London: Longman, Rees, Orne, et al.; 1830:4.

3. Cohen Y, Lavie O, Granovsky-Grisaru S, Aboulafia Y, Diamant Y. Bell palsy complicating pregnancy: a review. Obstet Gynecol Surv. 2000;55:184-188.

4. Robinerson JR, Pou JW. Bell's palsy-A predisposition of pregnant women. Arch Otolaryngol. 1972;95:125-129.

5. Deshpande AD. Recurrent Bell's palsy in pregnancy. J Laryngol Otol. 1990;1021:713714. 